\title{
Kaskat bir sistemin FTR sönümlenmeli kanallarda HQAM performans analizi
}

\section{Performance analysis of HQAM for a cascaded system over FTR fading channels}

\author{
Caner Özen1 (D), Mehmet Bilim²,*(D), \\ ${ }^{1}$ Nuh Naci Yazgan Üniversitesi, Fen Bilimleri Enstitüsü, 38090, Kayseri Türkiye \\ ${ }^{2}$ Nuh Naci Yazgan Üniversitesi, Elektrik Elektronik Mühendisliği Bölümü, 38090, Kayseri Türkiye
}

\begin{abstract}
Öz
$\mathrm{Bu}$ çalışmada, kaskat yapıya sahip bir sistemin dalgalanmalı iki 1şınlı (fluctuating two-ray, FTR) sönümlenmeye sahip kanallarda altıgen karesel genlik modülasyonu (hexagonal quadrature amplitude modulation, HQAM) analizi gerçekleştirilmiştir. Yapılan analizlerde ele alınan sistemin olasılık yoğunluk fonksiyonu (probability density function, PDF) kullanılmıştır. Sunulan hata analizlerinde hem yaklaşık hata ifadesi hem de matematiksel olarak daha sade bir analiz için asimptotik hata ifadesi türetilmiştir. Ele alınan sistemin performans değerlendirmeleri farklı kaskat bağlantı sayıları, sönümlenme parametre değerleri, yansıtıcı parametre değerleri ve modülasyon seviyeleri için gerçekleştirilmiştir. Yapılan analizler sonucunda elde edilen yaklaşık analitik ve asimptotik ifadelerin doğruluğu tam nümerik sonuçlarla gösterilmiştir.
\end{abstract}

Anahtar kelimeler: Kaskat FTR sönümlenmesi, Hata olasılığı analizi, HQAM tekniği

\section{Giriş}

Gelişen teknoloji ile birlikte gelecek nesil kablosuz haberleşme sistemlerinde kapsama alanının geniş olması beklenen gerekliliklerdendir. Kapsama alanının geniş tutulması için ise çok atlamalı (multi-hop) sistem modelleri ve kaskat (cascaded) sönümlü kanal türleri ortaya çıkmıştır. Kaskat sönümlü kanal, birbirine seri bağlı kanalların birleşimi gibi düşünülmektedir. Son 20 yıllık süreç içerisinde, kaskat sönümlü kanal modelleri ile ilgili literatürde birçok çalışma yapılmıştır [1-9]. [1]'de verilen çalışmada kaskat bağlantı düzenine sahip bir sistemin Nakagami- $m$ sönümlenmesinde istatistiksel analizleri sunulmuştur. [2]'de verilen çalışmada kaskat genelleştirilmiş $K$ sönümlenmesi ele alınırken, [3]'teki çalışmada bozuk radyo frekansı etkilerinin kaskat sönümlenmeli kanallarda incelemesi yapılmıştır. [4]'teki çalışmanın yazarları da hoyt sönümlenmesinin kaskat yapıda analizini gerçekleştirmişlerdir. Bir diğer çalışma olan [5]'te ise ultra yüksek frekanslı radyo frekansı tanımlama (ultra high frequency radio frequency identification, UHF RFID) sisteminin kaskat sönümlenmeli kanallardaki performans analizi yapılmıştır. Buna ilaveten, kaskat Rayleigh sönümlenmeli kanallarda araçlar arası çok atlamalı sistemin incelemesi [6]'daki çalışmada sunulmuştur. $\alpha-\mu$ sönümlenmesinde kaskat düzene sahip bir sistem analizi ise
Abstract

In this study, performance analysis of a cascaded system with hexagonal quadrature amplitude modulation (HQAM) is performed over fluctuating two-ray (FTR) fading channels. The probability density function of the considered system is employed in the analysis. In the presented error analysis, both the approximate error expression and the asymptotic error expression for a simpler mathematical analysis are derived. Performance evaluations of the considered system are carried out for different number of cascade connections, fading parameter values, reflective parameter values and modulation levels. Finally, the accuracy of the analytical and asymptotic expressions obtained as a result of the analyses is shown with exact numerical results.

Keywords: Cascaded FTR fading, Error probability analysis, HQAM technique

[7]'deki çalışmada yapılmıştır. [7]'nin yazarları bir başka çalışma olan [8]'de kompozit $\alpha-\mu$ sönümlenmesinde çok yollu yayılım ve gölgelemeli modellerin analizlerini gerçekleştirmişlerdir. Bir başka çalışma olan [9]'da ise kaskat $\alpha-\mu$ sönümlenmesinin güvenlik analizleri verilmiştir.

Öte yandan gelecek nesil kablosuz haberleşme sistemlerinden beklenilen gerekliliklerden bir diğeri ise yüksek veri hızıdır [10-13]. Bu beklentiyi de karesel genlik modülasyonu (quadrature amplitude modulation, QAM) tekniği yüksek oranda karşılayabilmektedir. QAM teknikleri içerisinde de farklı yıldız küme dağılımlarına sahip dikdörtgensel (rectangular), çapraz (cross) ve altıgen (hexagonal) türleri bulunmaktadır. HQAM tekniği bu modellerden öne çıkan adaylardandır ve yüksek veri iletiminde oldukça başarılıdır [14-17]. Bu sebeple, HQAM tekniğinin farklı haberleşme sistemlerine ait uygulamaları yapılmıştır [18-24]. [18]'de verilen çalışmada, kuvvetlendir ve aktar röleli bir ağın mükemmel olmayan kanal durum bilgisi ile HQAM hata analizi sunulmuștur. [19]'daki çalışmanın yazarı bir Gauss $Q$ fonksiyonu yaklaşıklığı sunmuş ve bunun uygulamasını HQAM analizi ile göstermiştir. Çok röleli bir ağın HQAM hata analizi ise [20]'de verilmektedir. $\eta$ - $\mu$ sönümlenmesinde iki kollu seçme birleștirme tekniğini kullanan bir sistemin HQAM hata analizi detaylı olarak [21]'deki çalışmada verilmektedir.

\footnotetext{
* Sorumlu yazar / Corresponding author, e-posta / e-mail: mbilim@nny.edu.tr (M. Bilim)

Geliș / Recieved: 10.12.2021 Kabul / Accepted: 28.01.2022 Yayımlanma / Published: 15.04.2022

doi: 10.28948/ngmuh.1034147
} 
[22]'deki çalışmada alternatif Rician sönümlenmesinde, içerisinde HQAM'in de bulunduğu farklı QAM teknikleri analiz edilmiştir. Verici kısımda iletim anteni seçmeli ve alıcıda ise en büyük oranlı birleştirme tekniğini kullanan çok girişli çok çıkışlı bir sistemin Weibull sönümlü kanallarda HQAM hata analizi ise [23]'te verilmiştir. [24]'deki yazarlar, Fisher-Snedecor $F$ sönümlenmesini dikkate alarak en büyük oranlı birleştirme tekniğini kullanan bir sistemin HQAM hata analizini sunmuşlardır.

Kaskat ve HQAM ile ilgili [1-9] ve [14-24]'te sunulan çalışmalar önemli olmasına rağmen hiçbiri kaskat dalgalanmalı iki ışınlı (fluctuating two-ray, FTR) kanallarda HQAM analizini ele almamıştır. [25-28]'de verilen çalışmalarda FTR kanalının band genişliği verimliliği açısından önemli bir yere sahip olacağı öngörüldüğünden ve milimetrik dalga iletişim için kullanılacağ 1 düşünüldüğünden bu kanal modeli öne çımaktadır. Kaskat FTR sönümlü kanal modeli [29] ise hem band genişliği verimliliğine hem de kaskat bağlantılı geniş kapsama alanına sahiptir. Tüm bu açıklamalar doğrultusunda, bu çalışmada, kaskat FTR sönümlü kanallarda HQAM tekniğinin hata değerlendirmesi literatürde ilk kez gerçekleştirilmiş̧ir. Bu sebeple, HQAM performansı için hata ifadesi türetilmiştir. Analizleri kolaylaştırmak için, türetilen ifadeler sadeleştirilerek asimptotik ifade elde edilmiştir. Türetilen hata ifadelerinin yardımıyla bazı yeni nümerik sonuçlar sunulmuştur. Verilen sonuçlarda farklı kaskat bağlantı sayısı, farklı FTR parametre değerleri ve farklı modülasyon seviyeleri ele alınmıştır.

\section{Sistem ve kanal modeli}

$\mathrm{Bu}$ çalışmada, kaskat bir sistem yapısı ele alınmaktadır. Literatürden de bilindiği üzere, kaskat bağlantı düzeni özellikle kablosuz bir sistemin kapsama alanının genişletilmesi için tercih edilmektedir. Bu sebeple, ele alınan sistemin kaskat yapıda olması tercih edilmiş̧tir. FTR sönümlenmeli kanallarda kaskat bir düzene ait sistemin alıcı taraftaki toplam alınan anlık işaret gürültü oranının (signal to noise ratio, SNR $(\gamma)$ ) olasılık yoğunluk fonksiyonu (probability density function, PDF) şu şekilde tanımlanmaktadır [29]:

$$
f_{\gamma}(\gamma)=\frac{1}{\prod_{l=1}^{N} \Gamma\left(m_{l}\right)^{j=0}} \sum_{j}^{\infty} \frac{t_{j}}{j !} \frac{\gamma^{k} e^{-\frac{\gamma}{2 \sigma^{2}}}}{\Gamma(j+1)\left(2 \sigma^{2}\right)^{j+1}}
$$

Burada $N$ kaskat düzendeki bağlantı sayısını, $m_{l} l$. kaskat bağlantısına ait sönümlenme parametresini belirtmektedir. Buna ilaveten $m_{l}$, FTR sönümlenmesinde Nakagami- $m$ dağılımlı sönümlenme parametresidir. $\Gamma(\cdot)$, Gama fonksiyonu, $\sigma^{2}$ ise gürültü varyansını belirtmektedir. Ayrıca, Denklem (1)'deki $t_{j}$ parametresi ise

$$
\begin{aligned}
& t_{j}=\sum_{k=0}^{j}\left(\begin{array}{l}
j \\
k
\end{array}\right) \sum_{h=0}^{k}\left(\begin{array}{l}
k \\
h
\end{array}\right) \sum_{n=0}^{w}(-1)^{2 n+2 h-k}\left(\frac{\Delta}{2}\right)^{2 n+2 h} \\
& \chi(n, w, 2 h-k) G_{1, N}^{N, 1}\left(\frac{1}{K} \prod_{l=1}^{N} m_{l} \mid \begin{array}{c}
1-(j+2 h-k+2 n) \\
m_{1}, m_{2}, \ldots, m_{N}
\end{array}\right)
\end{aligned}
$$

şeklinde tanımlanmaktadır. Burada $K$ parametresi $K=\left(V_{1}^{2}+V_{2}^{2}\right) / 2 \sigma^{2} \quad$ şeklinde tanımlanır ve FTR sönümlenmesinde bulunan $V_{1}$ ve $V_{2}$ iki adet yansitıcı bileşene bağlı olarak hesaplanan yansıtıcı oran parametresidir. $w$ seri üst sınır parametresidir ve $w \rightarrow \infty$ olduğunda sonsuz seriye yakınsamaktadır. $\Delta=\left(2 V_{1} V_{2}\right) /\left(V_{1}^{2}+V_{2}^{2}\right)$ olarak tanımlanı $V_{1}$ ve $V_{2}$ arasındaki ilişkiyi belirten bir parametredir. $G(\cdot)$ ise Meijer's $G$ fonksiyonudur. FTR sönümlenmesi için, $\bar{\gamma}$, ortalama SNR ifadesi

$$
\bar{\gamma}=\left(E_{b} / N_{0}\right) 2 \sigma^{2}(1+K)
$$

şeklindedir. Burada $E_{b}$ ve $N_{0}$ ise sırasıyla enerji ve güç spektral yoğunluklarını belirtmektedir. Ayrıca Denklem (2)'deki $\chi(\cdot)$ ise

$$
\chi(n, w, 2 h-k)=\frac{\Gamma(w+n)}{\Gamma(n+1) \Gamma(w-n+1)} \frac{w^{1-2 n}}{\Gamma(2 h-k+n+1)}
$$

olarak hesaplanmaktadır. Kaskat düzene sahip bir sistemin FTR sönümlü kanallardaki olasılıksal fonksiyonları bulunurken çalışma [30]'da sunulan difüzyon güçlü iki dalgalı (two-wave with diffuse power, TWDP) sönümlenmesinden $\quad f_{n F T R}=\int_{0}^{\infty} f_{T W D P}(x ; K=y K) f_{Y}(y) d y$ şeklinde faydalanılmıştır. Burada

$$
f_{Y}(y)=\frac{y^{-1}}{\prod_{l=1}^{N} \Gamma\left(m_{l}\right)} G_{0, N}^{N, 0}\left(y \prod_{l=1}^{N} m_{l} \mid \begin{array}{c}
- \\
m_{1}, m_{2}, \ldots, m_{N}
\end{array}\right)
$$

ve

$$
\begin{aligned}
f_{T W D P}(x)= & \exp (-K) \sum_{j=0}^{\infty} \frac{K^{j}}{j !} \frac{x^{j} \exp \left(-\frac{x}{2 \sigma^{2}}\right)}{\Gamma(j+1)\left(2 \sigma^{2}\right)^{j+1}} \\
& \sum_{k=0}^{j}\left(\begin{array}{l}
j \\
k
\end{array}\right)\left(\frac{\Delta}{2}\right)^{k} \sum_{l=0}^{k}\left(\begin{array}{l}
k \\
l
\end{array}\right) I_{2 l-k}(K \Delta)
\end{aligned}
$$

olarak tanımlanmaktadır. Denklem (5) ve (6), $f_{\text {nFTR }}=\int_{0}^{\infty} f_{\text {TWDP }}(x ; K=y K) f_{Y}(y) d y \quad$ ifadesinde $\quad$ yerine konulduğunda ve gerekli matematiksel işlemler 
gerçekleştirildiğinde Denklem (1)'de verilen PDF ifadesi elde edilmektedir [29]. Böylelikle bu çalışmada, PDF temelli bir yaklaşım kullanılmaktadır. Bu PDF ifadesi kuramsal ifadelerde kullanılıp, gerekli hata ifadesi analizleri gerçekleştirilmektedir.

\section{Performans analizleri}

Kablosuz haberleşme sistemleri için kullanılan genel hata ifadesi

$$
P_{e}=\int_{0}^{\infty} P(e \mid \gamma) f_{\gamma}(\gamma) d \gamma
$$

şeklindedir. Burada $P(e \mid \gamma)$ HQAM için toplamsal beyaz Gauss gürültüsü (additive white Gaussian noise, AWGN) şartlarında koşullu hata ifadesini ve $f_{\gamma}(\gamma)$ ise Denklem (1)'de verilen, ele alınan kaskat FTR sistem yapısı için PDF ifadesidir. Buradan hareketle bu çalışmada, HQAM analizinin yapılması hedeflendiğinden $P(e \mid \gamma)$ koşullu hata ifadesi [14]

$$
\begin{aligned}
P(\mathrm{e} \mid \gamma) & =K_{N N} Q(\sqrt{\xi \gamma})+\frac{2}{3} K_{N N} Q^{2}\left(\sqrt{\frac{2 \xi \gamma}{3}}\right) \\
& -2 K_{C N N} Q(\sqrt{\xi \gamma}) Q\left(\sqrt{\frac{\xi \gamma}{3}}\right)
\end{aligned}
$$

olarak verilmektedir. Burada $\xi, K_{N N}$ ve $K_{C N N}$ parametrelerinin değerleri $M$ modülasyon seviyesine göre değişim göstermektedir. Bu değerler Tablo 1'de verildiği gibidir. Denklem (8)'de verilen $P(e \mid \gamma)$ koşullu hata ifadesini matematiksel olarak daha basit bir hale getirmek için Gauss $Q$ fonksiyonunun $(Q(\cdot))$ tek terimli üstel fonksiyon yaklaşıklığı olan Chernoff üst sınırı ( $\left.Q(t)=\frac{1}{2} e^{-t^{2} / 2}\right)$ cinsinden yazıldığında aşağıda verilen yaklaşık ifade elde edilir:

$$
P(\mathrm{e} \mid \gamma) \approx \frac{K_{N N}}{2} e^{-\frac{\xi \gamma}{2}}+\frac{K_{N N}}{6} e^{-\frac{2 \xi \gamma}{3}}-\frac{K_{C N N}}{2} e^{-\frac{2 \xi \gamma}{3}}
$$

Buradan, Denklem (9) ve (1), (7)'de yerine konulursa bu çalışmada yapılmak istenilen kaskat bir yapıya sahip sistemin FTR sönümlü kanallarda hata olasılığı integrali elde edilir. Buna göre $P_{e}$

$$
\begin{aligned}
P_{e} & \approx \int_{0}^{\infty}\left[\frac{K_{N N}}{2} e^{-\frac{\xi \gamma}{2}}+\frac{K_{N N}}{6} e^{-\frac{2 \xi \gamma}{3}}-\frac{K_{C N N}}{2} e^{-\frac{2 \xi \gamma}{3}}\right] \\
& \times \frac{1}{\prod_{l=1}^{N} \Gamma\left(m_{l}\right)^{j=0}} \sum^{\infty} \frac{t_{j}}{j !} \frac{\gamma^{k} e^{-\frac{\gamma}{2 \sigma^{2}}}}{\Gamma(j+1)\left(2 \sigma^{2}\right)^{j+1}} d \gamma
\end{aligned}
$$

Tablo 1. $M$ değerlerine HQAM için $P(e \mid \gamma)$ 'deki parametreler [14]

\begin{tabular}{lccc}
\hline Modülasyon Seviyesi & $\xi$ & $K_{N N}$ & $K_{C N N}$ \\
\hline Optimum, $M=4$ & 1 & $5 / 2$ & $3 / 2$ \\
Optimum, $M=8$ & $32 / 69$ & $7 / 2$ & $21 / 8$ \\
Optimum, $M=16$ & $8 / 35$ & $33 / 8$ & $27 / 8$ \\
Optimum, $M=32$ & $512 / 4503$ & $75 / 16$ & $33 / 8$ \\
Optimum, $M=64$ & $8 / 141$ & $163 / 32$ & $75 / 16$ \\
Cift Elmas, $M=8$ & $4 / 9$ & $13 / 4$ & $9 / 4$ \\
Codex/ESE SP14.4, & $32 / 567$ & $81 / 16$ & $297 / 64$ \\
$M=64$ & $2 / 9$ & $33 / 8$ & $27 / 8$ \\
HEX, $M=16$ & $128 / 565$ & $17 / 4$ & $57 / 16$ \\
Üçgensel, $M=16$ & $2 / 9$ & $33 / 8$ & $27 / 8$ \\
TQAM, $M=16$ & $2 / 37$ & $161 / 32$ & $147 / 32$ \\
TQAM, $M=64$ & $2 / 149$ & $705 / 128$ & $675 / 128$ \\
TQAM, $M=256$ & $8 / 71$ & $75 / 16$ & $33 / 8$ \\
Düzenli TQAM, & & & \\
$M=32$ & & $75 / 16$ & $33 / 8$ \\
Alt optimum TQAM, & $8 / 71$ & & \\
$M=32$ & $8192 / 577595$ & $711 / 128$ & $171 / 32$ \\
Düzensiz TQAM, & & & \\
$M=256$ & & &
\end{tabular}

şeklindedir. Denklem (10) ile verilen ifade yeniden düzenlendiğinde

$$
\begin{aligned}
& P_{e} \approx \frac{1}{\prod_{l=1}^{N} \Gamma\left(m_{l}\right)^{j=0}} \sum^{\infty} \frac{t_{j}}{j !} \frac{1}{\Gamma(j+1)\left(2 \sigma^{2}\right)^{j+1}} \\
& \times\left[\begin{array}{l}
\int_{0}^{\infty}\left[\frac{K_{N N}}{2} e^{-\frac{\xi \gamma}{2}} \gamma^{k} e^{-\frac{\gamma}{2 \sigma^{2}}} d \gamma\right]+\int_{0}^{\infty}\left[\frac{K_{N N}}{6} e^{-\frac{2 \xi \gamma}{3}} \gamma^{k} e^{-\frac{\gamma}{2 \sigma^{2}}} d \gamma\right] \\
-\int_{0}^{\infty}\left[\frac{K_{C N N}}{2} e^{-\frac{2 \xi \gamma}{3}} \gamma^{k} e^{-\frac{\gamma}{2 \sigma^{2}}} d \gamma\right]
\end{array}\right]
\end{aligned}
$$

olarak yazılır. Denklem (11)'de birbiriyle aynı formatta olan üç tane integral bulunmaktadır. Bu integrallerin her biri [31, 3.381.4] yardımıyla çözülebilmektedir. Buna göre hata olasıliğ 1

$$
\begin{aligned}
& P_{e} \approx \frac{1}{\prod_{l=1}^{N} \Gamma\left(m_{l}\right)} \sum_{j=0}^{\infty} \frac{t_{j}}{j !} \frac{1}{\Gamma(j+1)\left(2 \sigma^{2}\right)^{j+1}} \\
& \times\left[\begin{array}{l}
\frac{K_{N N}}{2}\left(\frac{1}{2 \sigma^{2}}+\frac{\xi}{2}\right)^{-(j+1)} \Gamma(j+1) \\
+\frac{K_{N N}}{6}\left(\frac{1}{2 \sigma^{2}}+\frac{2 \xi}{3}\right)^{-(j+1)} \Gamma(j+1) \\
-\frac{K_{C N N}}{2}\left(\frac{1}{2 \sigma^{2}}+\frac{2 \xi}{3}\right)^{-(j+1)} \Gamma(j+1)
\end{array}\right]
\end{aligned}
$$

şeklinde türetilir. Denklem (12)'de verilen ifadeyi matematiksel olarak sadeleştirmek için Denklem (9)'daki $P(e \mid \gamma)$ koşullu hata ifadesindeki $Q^{2}(\cdot)$ 'li terimler etkisiz 
varsayıldığında ([22]'de sunulan çalışmadaki gibi) ve $j$ toplam indisi de 0 olarak belirlediğinde

$$
\begin{aligned}
P_{e}^{2} & \approx \frac{1}{\prod_{l=1}^{N} \Gamma\left(m_{l}\right)} \times \frac{t_{0}}{\Gamma(1) 2 \sigma^{2}} \\
& \times\left[\frac{K_{N N}}{2}\left(\frac{1}{2 \sigma^{2}}+\frac{\xi}{2}\right)^{-1} \Gamma(1)\right]
\end{aligned}
$$

olarak elde edilir. Denklem (13)'teki ifadenin yüksek SNR'daki davranışı analiz edilmek istenildiğinde $(\bar{\gamma} \rightarrow \infty)$ ifadedeki $2 \sigma^{2} \rightarrow 0$ olmaktadır. Buna göre hata ifadesinin asimptotik hali

$$
P_{e}^{\infty} \approx \frac{1}{\prod_{l=1}^{N} \Gamma\left(m_{l}\right)} \times \frac{t_{0}}{\left(2 \sigma^{2}\right)} \times \frac{K_{N N}}{2}\left(\frac{\xi}{2}\right)^{-1}
$$

olarak elde edilir.

\section{Bulgular}

Bu kısımda üçüncü bölümde yapılan analizler sonucunda elde edilen Denklem (12) ve (14) kullanılarak bazı nümerik sonuçlar verilmiştir. Elde edilen nümerik sonuçlar dört farklı senaryo ile sunulmaya çalışılmıştır. Bunlardan ilki, kaskat bağlı yol sayının değişimini incelemektedir. Şekil 1'de kaskat bağlı yollara ait $\left(m_{1}, m_{2}, m_{3}\right)$ Nakagami $m$ sönümlenme parametresi değerleri 5 olarak alınmış, $K=30$ ve $\Delta=0.5$ olarak belirlenmiştir. Modülasyon seviyesi optimum 8 ve sonsuz seri toplamdaki üst sınır ise 20 olarak alınmıştır. Ayrıca yine elde edilen hata ifadelerindeki $t_{j}$ katsayısındaki $\chi(n, w, 2 h-k)$ fonksiyonu için $w$ ise 20 olarak alınmıştır. Şekil 1'den de görüldüğü gibi, $N$ değeri arttıkça performans kötüleşmektedir. Ayrıca önceki bölümde sunulan analizler sonucunda elde edilen ifadelerin kullanılmasıyla ulaşılan analitik sonuçların, tam nümerik sonuçlarla iyi bir uyum içerisinde olduğu görülmektedir. Burada tam nümerik sonuçlar simülasyonlara tekabül etmekte ve analiz gerçekleştirilirken çözülen integrallerin tam sonuçlarını temsil etmektedir.

Şekil 2'de ise kaskat bağlantı sayısı $N=3$ olarak belirlenmiş, $K=30$ ve $\Delta=0.5$ olarak alınmıştır. Yine modülasyon seviyesi optimum $8, w=20$ ve sonsuz seri toplamdaki üst sınır ise 20 olarak kabul edilmiştir. Bu senaryoda ise farklı Nakagami- $m$ sönümlenme değerlerinin kıyaslaması yapılmaktadır. 3 bağlantılı kaskat yapıdaki bütün Nakagami- $m$ sönümlenme değerlerinin eşit ve sirasiyla $m_{1}=m_{2}=m_{3}=5$ ve $m_{1}=m_{2}=m_{3}=10$ olarak alınmıştır. Literatürdeki çalışma örneklerinde olduğu gibi, $m$ sönümlenme parametresinin değeri arttıkça hata değeri azalmakta ve sistemin performansı artmaktadır. $\mathrm{Bu}$ gerçeklik, Şekil 2'deki sonuçlarda da gözlemlenmektedir. $m_{1}=m_{2}=m_{3}=10$ olarak alındığı durumdaki hata performansının $m_{1}=m_{2}=m_{3}=5$ olarak alınan durumdaki hata performansından daha iyi olduğu görülmektedir. Özellikle, ortalama SNR değerinin $10 \mathrm{~dB}$ ve yukarısındaki değerlerde bu iyileşme daha çok göze çarpmaktadır. Bu senaryoda da analitik ve asimptotik sonuçlar ile tam simülasyon sonuçlarının uyumlu olduğu görülmektedir.

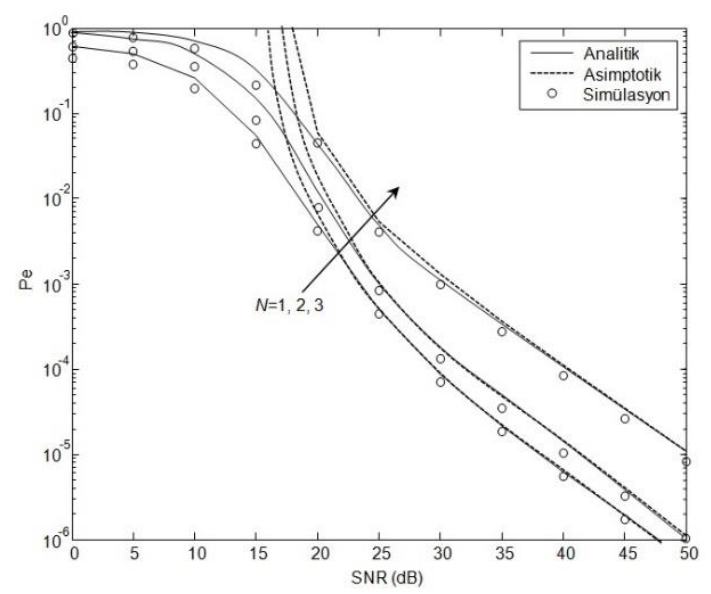

Şekil 1. Farklı kaskat bağlantı sayıları için hata performans kiyaslaması

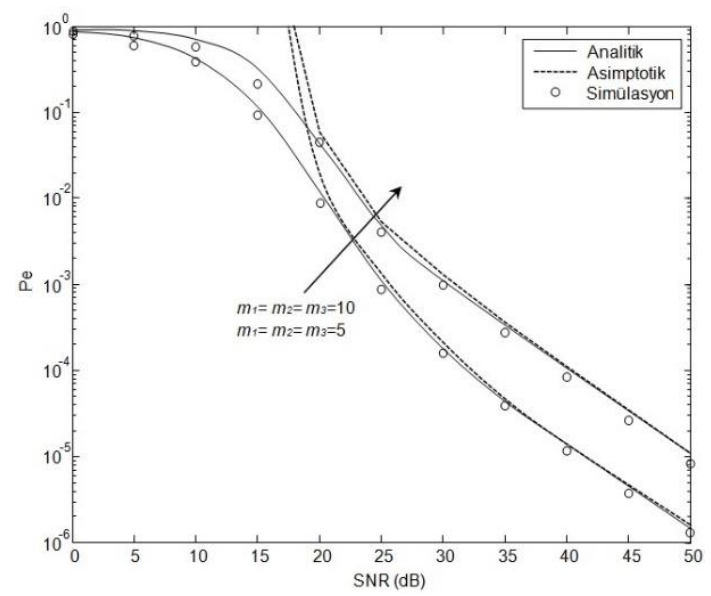

Şekil 2. Kaskat bağlantılardaki farklı $m$ değerleri için hata performansı kıyaslaması

Şekil 3'teki senaryoda FTR sönümlü kanallarda iki yansıtıcı bileşene bağlı olarak hesaplanan yansitıcı oran parametresi $K^{\prime}$ nın sırasıyla 15 ve 30 olduğu değerlerin hata performans kıyaslaması sunulmuştur. Burada yine kaskat bağlantı sayısı $\quad N=3, \quad \Delta=0.5, \quad m_{1}=m_{2}=m_{3}=5$, modülasyon seviyesi optimum $8, w=20$ ve sonsuz seri toplamdaki üst sınır ise 20 olarak alınmıştır. Elde edilen sonuçlarda literatürdeki FTR sönümlü kanallarda yapılan sonuçlara benzer şekilde, $K$ değeri arttıkça sönümlenme etkisinin azaldığı ve buna paralel olarak incelenen sistemin hata performansının iyileştiği açık bir şekilde görülmektedir. Şekil 2'deki sonuçlara benzer şekilde, buradaki senaryoda da ortalama SNR'nin 10 dB'yi geçtikten sonra bahsedilen bu iyileşme belirgin şekilde dikkati çekmektedir. Şekil 3'teki sonuçlar gözönüne alındığında analitik ve asimptotik sonuçların yine tam simülasyon sonuçlarıyla iyi bir uyum içerisinde olduğu gözlemlenmektedir. 


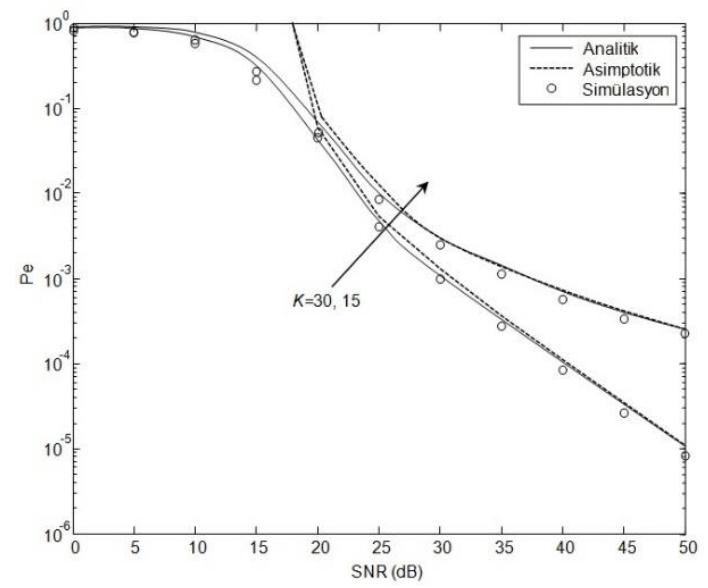

Şekil 3. Farklı $K$ değerleri için hata performans kıyaslamas1

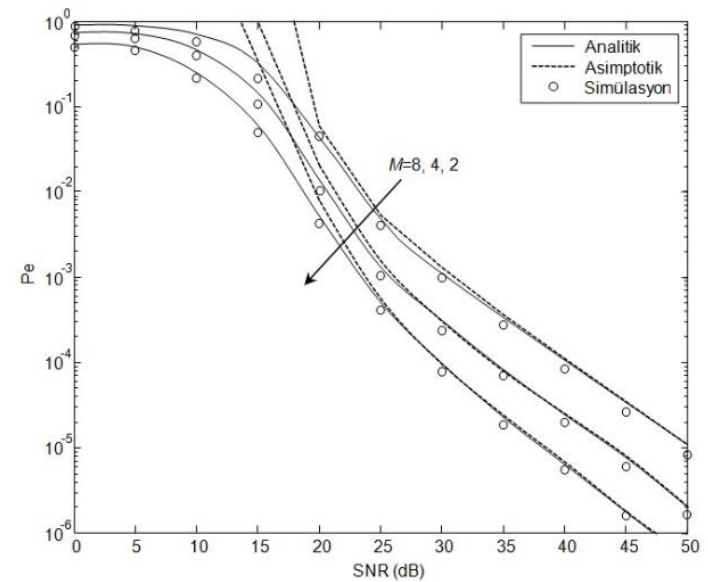

Şekil 4. Farklı modülasyon seviyeleri için hata performansı kıyaslaması

$\mathrm{Bu}$ çalışmada sunulan son senaryo olan Şekil 4'te ise farklı modülasyon seviyelerinin kıyaslaması verilmektedir. Burada da kaskat bağlantı sayısı $N=3, \Delta=0.5$, $m_{1}=m_{2}=m_{3}=5, K=30, w=20$ ve sonsuz seri toplamdaki üst sınır ise 20 olarak alınmıștır. Farklı modülasyon seviyesine sahip (2-HQAM, 4-HQAM ve 8-HQAM) kaskat sistemlerinin beklenildiği gibi hata performanslarının da farklı olduğu görülmektedir. Literatürdeki çalışmalardan da bilindiği üzere, düşük modülasyon seviyesine sahip sistemin hata performansının daha üstün olması beklenmektedir. $\mathrm{Bu}$ durum, bu senaryoda açık bir şekilde görülmektedir. Düşük modülasyon seviyesi olduğunda komşu bitlerin birbirlerine olan yakınlığı daha az olacak ve bu da algılamada daha az hata yapma olasılığını ortaya çıkaracaktır. Bu sebeple, bu senaryoda en iyi hata performansı modülasyon seviyesi 2 olan sisteme ait olup, en kötü hata performansı ise modülasyon seviyesi 8 olan sisteme aittir. Daha önceki senaryolarda olduğu gibi, burada da hata analizleri sonucunda türetilen analitik ve asimptotik eşitlikler kullanılarak elde edilen sonuçların tam simülasyonlarla uyumlu olduğu görülmektedir. Şekil 1-4'te verilen sonuçlardaki bu uyum yapılan hata analizlerinin doğruluğunu göstermektedir.

\section{Sonuçlar}

Bu çalışmada, HQAM kullanan kaskat bağlantı düzenine sahip bir sistemin FTR sönümlenmeli kanallarda hata analizleri gerçekleştirilmiştir. Hata analizleri yapılırken matematiksel olarak kullanım kolaylığından dolayı Chernoff Gauss $Q$ fonksiyonu yaklaşıklığından ve sistemin PDF ifadelerinden faydalanılmıştır. Yapılan analizleri matematiksel olarak daha basit bir formda elde etmek için asimptotik ifade de türetilmiştir. Ele alınan sistem için temel parametreler olan kaskat bağlantı sayısı, sönümlenme parametresi, yansitıcı oran parametresi ve modülasyon seviyesi gibi değerlerin sistem üzerindeki etkileri gösterilmeye çalışılmıştır. Literatüre uygun bir şekilde bahsi geçen parametre değişimlerinin etkileri ele alınan kaskat sistem üzerinde de görülmüştür. Yapılan analizlerin geçerliliği ise analizlerdeki ifadelerin tam nümerik sonuçlarının kıyaslaması ile verilmiștir. Son olarak FTR yeni bir sönümlenme modeli olduğu için gelecekte, iki kollu, çok atlamalı iletişim, uyarlanabilir akıllı yüzey destekli iletişim gibi farklı sistem modellerine adapte edilerek yeni çalışmalar ortaya konulabilir.

\section{Çıkar çatışması}

Yazarlar çıkar çatışması olmadığını beyan etmektedir

Benzerlik oranı (iThenticate): $\% 11$

\section{Kaynaklar}

[1] G. K. Karagiannidis, N. C. Sagias, and P. T. Mathiopoulos, $N^{*}$ Nakagami: A novel stochastic model for cascaded fading channels. IEEE Transactions on Communications, 55 (8), 1453-1458, 2007. doi: 10.1109/TCOMM.2007.902497.

[2] K. Peppas, F. Lazarakis, A. Alexandridis, and K. Dangakis, Cascaded generalized- $K$ fading channel. IET Communications, 4 (1), 116-124, 2010. doi: 10.1049/iet-com.2009.0107

[3] A.-A. A. Boulogeorgos, P. C. Sofotasios, B. Selim, S. Muhaidat, G. K. Karagiannidis, and M. Valkama, Effects of RF impairments in communications over cascaded fading channels. IEEE Transactions on Vehicular Technology, 65 (11), 8878-8894, 2016. doi: 10.1109/TVT.2016.2516901.

[4] N. Hajri, N. Youssef, T. Kawabata, M. Pätzold, and W. Dahech, Statistical properties of double Hoyt fading with applications to the performance analysis of wireless communication systems. IEEE Access, 6, 19597-19609, 2018. doi: 10.1109/ACCESS.2018.2820746.

[5] A. Bekkali, S. Zou, A. Kadri, M. Crisp, and R. V. Penty, Performance analysis of passive UHF RFID systems under cascaded fading channels and interference effects. IEEE Transactions on Wireless Communications, 14 (3), 1421-1433, 2015. doi: 10.1109/TWC.2014.2366142.

[6] Y. Alghorani, G. Kaddoum, S. Muhaidat, S. Pierre, and N. Al-Dhahir, On the performance of multihopintervehicular communications systems over $n *$ Rayleigh fading channels. IEEE Wireless 
Communications Letters, 5 (2), 116-119, 2016. doi: 10.1109/LWC.2015.2505308.

[7] E. J. Leonardo and M. D. Yacoub, Product of $\alpha-\mu$ variates. IEEE Wireless Communications Letters, 4 (6), 637-640, 2015. doi: 10.1109/LWC.2015.2476791.

[8] E. J. Leonardo and M. D. Yacoub, The product of two $\alpha-\mu$ variates and the composite $\alpha-\mu$ multipathshadowing model. IEEE Transactions on Vehicular Technology, 64 (6), 2720-2725, 2015. doi: 10.1109/TVT.2014.2342611.

[9] L. Kong, G. Kaddoum, and D. B. da Costa, Cascaded $\alpha-\mu$ fading channels: reliability and security analysis. IEEE Access, 6, 41978-41992, 2018. doi: 10.1109/ACCESS.2018.2833423.

[10] M. K. Simon and M-S. Alouini, Digital communication over fading channels. 2nd ed. Hoboken, New Jersey, USA: IEEE: John Wiley \&Sons, Inc., 2005.

[11] J. Rischke, P. Sossalla, S. Itting, F. H. P. Fitzek and M. Reisslein, 5G campus networks: a first measurement study. IEEE Access, 9, 121786-121803, 2021. doi: 10.1109/ACCESS.2021.3108423.

[12] L. -H. He, Y. -L. Ban and G. Wu, Dual-band quadpolarized transmitarray for $5 \mathrm{G} \mathrm{mm}$-wave application. IEEE Access, 9, 117520-117526, 2021. doi: 10.1109/ACCESS.2021.3106949.

[13] M. Bilim and N. Kapucu, Average symbol error rate analysis of QAM schemes over millimeter wave fluctuating two-ray fading channels. IEEE Access, 7, 105746-105754, 2019.2 doi: 10.1109/ACCESS.2019.2932147.

[14] L. Rugini, Symbol error probability of hexagonal QAM. IEEE Communications Letters, 20 (8), 15231526, 2016. doi: 10.1109/LCOMM.2016.2574343.

[15] M. K. Simon and J. G. Smith, Hexagonal multiple phase-and amplitude-shift-keyed signal sets. IEEE Transactions on Communications, 21 (10), 1108-1115, 1973. doi: 10.1109/TCOM.1973.1091549.

[16] S. Hosur, M. F. Mansour, and J. C. Roh, Hexagonal constellations for small cell communication. in Proc. IEEE Global Commun. Conf. (GLOBECOM), sayfa 3270-3275, Atlanta, GA, USA, 2013. doi: 10.1109/GLOCOM.2013.6831576.

[17] J. Lee, D. Yoon, and K. Cho, Error performance analysis of $M$-ary $\theta$-QAM. IEEE Transactions on Vehicular Technology, 61 (3), 1423-1427, 2012. doi: 10.1109/TVT.2012.2186835.

[18] P. K. Singya, N. Kumar and V. Bhatia, Impact of imperfect CSI on ASER of hexagonal and rectangular QAM for AF relaying network. IEEE Communications Letters, 22 (2), 428-431, 2018. doi: 10.1109/LCOMM.2017.2778153.

[19] D. Sadhwani, R. N. Yadav and S. Aggarwal, Tighter bounds on the Gaussian $Q$ function and its application in Nakagami- $m$ fading channel. IEEE Wireless Communications Letters, 6 (5), 574-577, 2017. doi: 10.1109/LWC.2017.2717907.
[20] N. Kumar, P. K. Singya and V. Bhatia, ASER analysis of hexagonal and rectangular QAM schemes in multiple-relay networks. IEEE Transactions on Vehicular Technology, 67 (2), 1815-1819, 2018. doi: 10.1109/TVT.2017.2758028.

[21] Bilim, M., Dual-branch SC wireless systems with HQAM for beyond $5 \mathrm{G}$ over $\eta-\mu$ fading channels. Peerto-Peer Networking and Applications, 14, 305-318, 2021. doi: 10.1007/s12083-020-00946-X.

[22] Bilim, M., Different QAM Schemes analyses for ARS fading channels. Transactions on Emerging Telecommunications Technologies, 32 (1), e4119, 2021. doi: 10.1002/ett.4119.

[23] Bilim M., Altıgen kareleme genlik modülasyonu kullanan MIMO TAS/MRC sistemlerin Weibull sönümlenmeli kanallardaki hata analizi değerlendirmesi. EEMKON 19, Elektrik Elektronik Mühendisliği Kongresi, sayfa 410-413, 2019, Türkiye.

[24] C Özen, M Bilim HQAM Analysis of MRC Diversity Systems over Fisher-Snedecor $F$ Channels. 29th Signal Processing and Communications Applications Conference, sayfa 1-4, 2021, Türkiye. doi: 10.1109/SIU53274.2021.9477903.

[25] J. M. Romero-Jerez, F. J. Lopez-Martinez, J. F. Paris and A. J. Goldsmith, The fluctuating two-ray fading model: statistical characterization and performance analysis. IEEE Transactions on Wireless Communications, 16 (7), 4420-4432, 2017. doi: 10.1109/TWC.2017.2698445.

[26] J. Zhang, W. Zeng, X. Li, Q. Sun and K. P. Peppas, New results on the fluctuating two-ray model with arbitrary fading parameters and its applications. IEEE Transactions on Vehicular Technology, c. 67, no. 3, s. 2766-2770, 2018. doi: 10.1109/TVT.2017.2766784.

[27] W. Zeng, J. Zhang, S. Chen, K. P. Peppas and B. Ai, Physical layer security over fluctuating two-ray fading channels. IEEE Transactions on Vehicular Technology, $67 \quad$ (9), 8949-8953, 2018. doi: 10.1109/TVT.2018.2842126.

[28] J. Zheng, J. Zhang, G. Pan, J. Cheng and B. Ai, Sum of squared fluctuating two-ray random variables with wireless applications. IEEE Transactions on Vehicular Technology, 68 (8), 8173-8177, 2019. doi: 10.1109/TVT.2019.2920533.

[29] O. S. Badarneh and D. B. da Costa, Cascaded fluctuating two-ray fading channels. IEEE Communications Letters, 23 (9), 1497-1500, 2019. doi: 10.1109/LCOMM.2019.2926982.

[30] G. D. Durgin, T. S. Rappaport, and D. A. de Wolf, New analytical models and probability density functions for fading in wireless communications. IEEE Transactions on Communications, 50 (6), 1005-1015, 2002. doi: 10.1109/TCOMM.2002.1010620.

[31] I. Gradshteyn and I. Ryzhik, Table of Integrals, Series and Products. 6th ed. New York, NY, USA: Academic, 2000. 